\title{
MULTISCALE MODELING OF COMPOSITE LAMINATES DELAMINATION VIA COMPUTATIONAL HOMOGENIZATION
}

\author{
Lu Ke*, Frans van der Meer \\ Faculty of CEG, Delft University of Technology, Delft, The Netherlands \\ *1.ke@tudelft.nl
}

The material performance of composites is challenging to understand and predict, as a consequence of complex underlying heterogeneous microstructure. As a key weakness of laminated composites, delamination needs to be addressed adequately prior to the design on higher structural level. Numerical models $[1,2]$ have been proposed to carry out virtual testing of this failure process. Cohesive laws are commonly adopted to describe the crack growth where experimentally observed mode-ratio dependence of the fracture energy is included through phenomenological relations. This fracture energy is a crucial input; its accuracy under general load conditions is essential for reliability of computational models.

Multiscale modeling [3] is a promising approach to provide more insight into energy dissipation during crack growth. Processes such as matrix plasticity and fibre/matrix interface debonding are best described on the microscale. Through computational homogenization, microscale energy dissipation is upscaled to the mesoscale where cohesive tractions are computed on-the-fly without prior knowledge of fracture toughness as in classical phenomenological relations.

In this work, delamination of unidirectional laminated composites is simulated by a computational homogenization model which is designed to tackle fracture on both scales. On the mesoscale XFEM is adopted to simulate the initiation and propagation of delamination cracks while on the microscale a model that inserts cohesive elements between neighboring elements on the fly is used. For the matrix, a polymer/plasticity model is used. The amount of energy dissipation from different sources is investigated and the mode-ratio dependence from the simulations is compared to experimental observations.

\section{References}

[1] F. P. van der Meer and L. J. Sluys (2013) A numerical investigation into the size effect in the transverse crack tension test for mode II delamination. Compos. Part A Appl. Sci. Manuf., 54, 145-152.

[2] A. Turon, E. V. González, C. Sarrado, G. Guillamet, and P. Maimí (2018) Accurate simulation of delamination under mixed-mode loading using a cohesive model with a modedependent penalty stiffness. Compos. Struct., 184, 506-511.

[3] E. Svenning, F. Larsson, and M. Fagerström (2019) A two-scale modeling framework for strain localization in solids: XFEM procedures and computational aspects. Comput. Struct., 211, 43-54. 